\section{达西安娜・菲萨克对中国现 当代文学作品的译介研究}

\section{Taciana Fisac's \\ Translation of \\ Chinese Modern and \\ Contemporary Literature}

朱旭晨

燕山大学文法学院

秦皇岛 河北 中国, 066004 zhuxuchen16@163.com

Yanshan University

College of Humanities and Law Qinhuangdao, Hebei China 066004
文学国际化的历程一方面彰显着源语文学 创作的成就, 一方面见证着目的语读者了解 源语国的需求, 同时, 也从一个侧面反映着 国际战略与国际关系的发展变化。中国现当 代文学作品的西语译介始于1949年, 但直到 1978年才出现译自中文原版的译作。达西安 娜 - 菲萨克正是西班牙第一批从事中西文学 对译的译者。从1982年翻译鲁迅的《立论》 与《这样的战士》迄今, 其全部译作均直接 译自中文原版，在译介对象难度增大的同 时, 译作与目的语读者的接受习惯越来越靠 近，基本形成了个人的翻译思想与方法。既 重视译者对源语文学作品的整体把握与鉴赏 能力, 又特别强调译者目的语的文学表达与 文化传递水准; 既注重对源语文学作品叙事 方法与风格的尊重，更顾念目的语读者的接 受心理与表达方式。其翻译实践丰富了翻译
The internationalization of literature manifests achievement of source language literature and witnesses the target language readers' requirement of understanding the source language country. Moreover, it reflects the developments and changes on international strategies and relationships. The translation of Chinese literature to Spanish began from 1949, but the first translation from Chinese original edition arose in 1978. Taciana Fisac is one of the first Chinese-Spanish translators in Spain. All her translations were translated from Chinese original edition since Lu Xun's “Argument" and "Such soldiers" in 1982. Although it is much more difficult, this translation mode is closer to target reader's habit, and forms personal

* 朱旭晨, 复旦大学文学博士, 燕山大学文法学院教授, 曾职于马德里自治大学东亚研究中心。研究方向 为中国现当代文学、海外华文文学、传记文学等。 
达西安娜・菲萨克对中国现当代文学作品 的译介研究

30

文学理论, 为中国现当代文学作品的国际化 做出了不可磨灭的贡献。

[关键词] 翻译实践; 归化; 异化; 接受美 学; 达西安娜・菲萨克
Taciana Fisac's Translation of Chinese Modern and Contemporary Literature

translation method. This methodology requires translator's overall grasp and connoisseurship of source literature as well as literature expression and culture delivery ability in target language. Besides, it not only pays attention to source language literatures narrative style, but also considers target language reader's reception psychology and expression. Taciana Fisac's translation enriched theory of literature translation, and made significant contribution for internationalization of Chinese modern and contemporary literature.

Key Words: translation practice; domestication; foreignization; reception aesthetics; Taciana Fisac.

从文学翻译到翻译文学, 语序的变化彰显出文学翻译的蓬勃发展与新 学科的逐渐形成, 贾植芳 (2003) “认为, 中国现代文学的历史, 除理论 外, 就作家作品而言, 应由小说、诗歌、散文、戏剧和翻译文学五个单元组 成。”回顾中国新文学的形成与发展及 20 世纪 80 年代以来文学场景、创作实 绩的变化与国际影响力的提升, 笔者亦赞同这一说法。对中外文学场域的译 介活动进行检视于是成为学科发展的首要举措。

对比 20 世纪以来中国翻译文学蔚然大观的景象, 反向输出（即中国现当 代文学作品的外译）, 状况如何? 就答案所需付出的巨大工作量而言, 这显 然是一项颇为庞大的工程, 有待于学者们的共同努力。这里, 笔者仅就达西 安娜 - 菲萨克对中国现当代文学作品的西译（狭义的西译，专指西班牙语译 介, 笔者注) 展开论述。

\section{1. 译介概述}

中国现当代文学作品的西语译介始于1949年, 即Rosa María Topete译自 英文版的谢冰荣的《女兵自传》（Autobiografía de una muchacha china）。 译自中文原版的译作直到1978年才出现, 即西班牙资深汉学家、藏学家毕隐 崖（Iñaki Preciado Idoeta）翻译的鲁迅的《呐喊》（Grito de llamada）。 需要说明的是, 1975年Joaquín Horta翻译出版了《毛泽东诗歌》 (Zedong Mao: Poemas），但鉴于国内外文学史家极少将毛泽东列入作家诗人行列, 故此处存疑, 以备商榷。 
达西安娜・菲萨克正是西班牙第一批从事中西文学翻译的学者。1982年, 她在Nuevo indice杂志 (《新迹象》) 首次发表译作La argumentación（《立 论》) 、Un soldado así ( 《这样的战士》) 。之后, 陆续翻译出版了La familia Kao (《家》1985) 、La blusa roja sin botones（《没有纽扣的红祄衫》1989）、Chen Huansheng va a la ciudad ( 《陈焕生进城》1989) 、La fortaleza asediada（《围 城》1992)、Viaje a Xibanya: Escritores chinos cuentan España（《西行西行: 中国作家西班牙纪行》2010）、El enólogo volador（《飞行酿酒师》2016）、Los cuatro libros（《四书》2016）、Caían chuzos de punta（《大雨如注》2017）、El pequeño apéndice（《小尾巴》2018）以及全本的Intramuros-Especial China（第 37期）（《在城墙内-中国特别报导》2013），其中包括阿莱、毕飞宇等20位当 代作家的传记、自传和短篇小说等。可贵的是达西安娜 - 菲萨克的全部译作均 直接译自中文原版。其累计三百余万字的案头翻译工作全靠业余时间进行。据 此, 我们认为, 对中国的关注与译介伴她走过青春年华后, 她已将这项工作视 为自己的天赋职责。其突出贡献获得了中国政府的赞赏，2012年8月达西安娜 菲萨克获第六届中华图书特殊贡献奖。

\section{2. 菲萨克的翻译思想}

学者王向远 (2015) 提出 “翻译思想即翻译的思想, 是研究和思考翻译 问题而产生的有创意的观点主张或理论建构。”通常来说, 翻译家未必都有 系统的翻译思想，但多年的翻译实践总会催使其对某些问题展开思考，日积 月累，或可形成既 “有严谨的逻辑、有理论的深度”，又有 “理论想象力和 鲜活的生命体验”，同时 “具有思考与表达的独特性、创新性、启发性与耐 用性” 的思想。作为大学教授、博士生导师、中心主任, 达西安娜 - 菲萨克 的日程安排十分紧张，无暇系统整理关于翻译问题的见解，但其翻译实践及 仅有的几次会议发言还是为我们提供了一些思路和启发, 或可代为总结一二。

\section{1 从选本及其导读考察}

面对洋洋大观的中国现当代文学作品, 再高效的译者也不禁要望而生畏, 于是, 对作品的选择就变得极为重要。选出代表中国整体创作实力的作家, 及 该作家富有个人特色并能代表其创作水准，同时又是译者个人喜爱，也符合目 的语读者的审美品味的作品, 是所有译者的首要任务。除去个别作为两国政府 间的文化交流任务必须完成外，仔细审阅达西安娜 - 菲萨克翻译的长篇短制， 即可发现其作品选择的标准，亦能体悟到其逐渐明晰的翻译思想。

达西安娜 - 菲萨克对中国文学作品的翻译, 涉及到整个现当代阶段。就 现代文学而言, 她选择翻译的作家虽然只有三位, 即鲁迅、巴金、钱钟书, 却刚好覆盖了 20 年代、30年代、 40 年代, 每个十年一位。《野草》、《家》、 《围城》虽是中国学者耳熟能详的作品, 但在时年二三十岁的达西安娜眼 中, 无疑是大大小小的险山峻岭。作为译者, 她在坚持不解地下了番苦功 后, 渐入佳境。其对作家作品的深刻理解和把握, 主要体现在她所写的西语 
达西安娜・菲萨克对中国现当代文学作品 的译介研究
Taciana Fisac's Translation of Chinese Modern and Contemporary Literature

版作家简介及作品导读中。仔细阅读, 笔者发现其中亦蕴藏着她在翻译过程 中逐渐形成的高规格选择标准。

对鲁迅，菲萨克（1982）强调其作品的批判性和巧妙的构思，认为 《立论》和《这样的战士》展示了中国人的奇特智慧: 简短、深刻、夸张、 诗意、幽默、悲哀。在关于巴金及其作品的介绍中，她简洁地追溯了自巴金 出生的1904年至80年代的中国历史，包括辛亥革命、五四文学运动、新中国 建立及文化大革命。历史的追溯与巴金的生平相交融, 可以更好地帮助西班 牙读者理解作家生活的时代及其创作。显然，菲萨克（1989）对于巴金的经 历及创作都有较为全面的把握，对他的笔名由来及创作缘起等解释得清晰明 了，对影响巴金的时代、家庭、安那其主义（即无政府主义）均有点到为止 的介绍。菲萨克（1989）在封底以极为简约凝练的语言概括出 “高老太爷是 家族的绝对领导，他的权威基于旧政权下的家族制度：孝顺和仪式。他认为 钱是万能的，从没想过年青人会有自己的想法……他不知道他的专制将把他 的孙子变成革命者”。而西语版的《围城》，无译者序或是译后记，只有介 绍作者的封二和介绍作品的封底。说到作品, 菲萨克 (2009) 认为《围城》 是20世纪中叶中国现代文学的巅峰之作。作品通过主角方鸿渐生活场景的变 迁绘制了一幅高雅而智慧的图画, 壮丽辉煌, 并极富讽刺意味。它借助对陈 词滥调和陈旧观念的嘲讽，向西方读者无情地揭开了古老文化的神秘面纱， 揭示了数千年华夏文明的颓废、知识界和大学的空虚、渗透于社交生活中的 西化取向。简而言之, 它既类似于克服日常生活障碍和不适的流浪汉小说, 又是对传统和习俗的强烈讽刺, 是一部精彩的悲喜剧, 也是一幅战争期间的 国家全景图。由此可见，鲁迅的犀利深刻、悲哀与幽默，巴金的 “我控诉” 及颠覆旧家族制度的呼喊，钱钟书将传统文明和知识分子置于战争时期的特 定场景而进行的无情讽刺, 都是她所欣赏并乐于译介的。民族性、时代感, 以及作品自身呈现出的思想深度和语言鬼米力, 无疑是她选择作品时着重考虑的。

当代文学是她近年来翻译的重点, 涉及的作家作品较为广泛。她较早 接触和翻译的是铁凝、高晓声等人的作品，后来又有毕飞宇、曹文轩等人的 作品, 历时较长。在《飞行酿酒师》和《没有纽扣的红衬衫》中, 除了那些 必备的客观介绍外，菲萨克（2016）她认为铁凝的作品直接坦率，善于处理 那些深陷于加速发展的社会变革中的男女，及其日常生活和问题，持续关注 人们的生活, 特别是女性。不仅如此, 她还能以冷静和批判的目光凝视中 国社会的巨大转变和正在浮出水面的新问题。在《没有纽扣的红祄衫》译者 序中，菲萨克（1989）较为详细地介绍了当代中国的政治运动，对 “大跃 进”、“文革”、“上山下乡” 等知识分子 “再教育” 以及1976年毛泽东逝 世、“四人帮” 倒台后中国 “四化” 目标的确立、改革开放起步等都做出了 符合实际的解说。这些背景性的介绍和交代有助于西班牙读者把握和理解 作品中故事的发生和人物的行为, 为此, 她还举例说 “一个 16 岁的女孩如 果说 “吻” 这个词就会被认为是不规矩的。” “传统上，中国夫妻之间充满 了禁忌，在文革期间更严重，考虑到政治斗争之外的感觉几乎是不可思议 的。”这些文字呼应着译者序开篇所言 “这本只是简单地书写生命及两个人 
的情怀而无关政治、社会阶层和经济的小说, 无疑在中国多年的创作和出版 史上是不可能发生的事情”, 以及封底所云 “该作品让我们近距离审视中国 社会, 它有那么多与我们酷似又很不同的事情。” 在介绍高晓声时, 菲萨克 （1989）强调其作品的通俗易懂和讽刺性, 认为他擅长分析中国当代农村社 会问题和农民的心理, 因为他生长及受教育的环境都在农村。对毕飞宇, 菲 萨克（2017）则重点介绍其屡获奖项及作品被译介和改编的情况, 并强调毕 飞宇关于创作的 “化学” 性生成观, 即相同的事实和情况人们的反应完全不 同, 即便是同一个作家, 在他生命中的不同时刻面对同样情形反应也是不同 的。每部小说都既与作家创作的时代相呼应, 又显示了时代赋予自己的提炼 能力。对毕飞宇来说, 这同想象力一样重要。他的写作充满了情感, 他试图 理解那些渴望获得文学生活的活在当下的普通人, 这表现出他对他人、生活 和世界的态度。他的作品叙事清晰直接, 与口语非常接近, 这使他成为海内 外最著名的小说家之一。所有翻译中, 耗时最长的要数阎连科的《四书》, 它几乎占用了菲萨克 6 个年头的时间。读过港台版《四书》的人都知道, 仅 就语言来说, 《四书》的翻译难度已堪称 “蜀道”。那种白话文杂糅着文言 文、方言土语和圣经句式的语言, 即便是中国人, 读起来也是蛮吃力的。但 是, 她十分推崇这本书, 很重视它的翻译。为此, 她特别写了篇《四书导 读》, 放在前面, 这在她的译著中是极为罕见的。在菲萨克（2016）看来,

《四书》 “注定要成为当代文学的经典之作”。在导读中, 她首先指出阎连 科的创作灵感主要源于自身的经历, 那些看似离奇而疯狂的举动、以及那些 微小的细节并不是作家想象力的产物。接下来, 她就为不了解当代中国政治 运动的西班牙读者详细介绍了《四书》故事发生的时代背景。先是从1956年 百花齐放百家争鸣的 “双百方针” 到1957年的反右运动, 这致使数十万专业 人士和学者成为 “右派” 、被贴上 “反革命” 的标签, 接受再教育。接着 就是1958年极度浮夸造假的 “大跃进” 和随之而来的造成三千多万人死 亡的 “自然灾害”（1959-1961），之后又是被称之为 “十年动乱” 的 “文 革”（1966-1976）。并解释了诸如 “红卫兵”、“红宝书” 等西班牙读者未 曾听闻的词语。随后, 她写到, “这里叙述的故事应等同于其他人类历史上 惨痛的大屠杀, 因为它以数千万人的死亡而告终。” 在这些当代历史大事件 的叙述后, 作为文学教授, 她又对 《四书》的创作做出了艺术方面的阐释, 从中国古代的 “四书” 到阎连科的 “四书”，并提到书名内涵的双重乃至多 重性。菲萨克（2016）肯定阎连科的叙述，认为以他的文笔、他对社会现实 独具慧眼而深刻犀利的分析, 没有人可以对他的作品无动于衷。他的作品再 现了那个复杂的、重要的、又难以说清楚的引人注目的时代。如同 20 世纪初 期那些中国最伟大的作家一样, 阎连科用笔践行着作家的社会责任。最后, 她详细阐释了阎连科的创作方法和风格, 对阎连科所谓的 “神实主义”、作 品的诗意营造手法及创作中的戏仿成分等都做出了清晰的界说。

通过对其翻译的作家作品选择情况及介绍和导读的分析, 我们发现她的 选择标准是内容与艺术兼顾, 她既注重作家的社会影响力, 同时也非常欣赏 有思想深度和独特风格、富有时代感或反映重大历史及现实问题的作品。 
达西安娜・菲萨克对中国现当代文学作品 的译介研究
Taciana Fisac's Translation of Chinese Modern and Contemporary Literature

\section{2 从会议发言中追寻}

作为文学走出去的举措之一, 中国作家协会每两年举行一次汉学家文学 翻译国际研讨会。除第一届外（2010年8月，北京，“中国文学翻译经验与 建议”一一笔者注），菲萨克应邀参加了第二三四届研讨会，并做了大会发 言。梳理她三届研讨会的发言, 我们可以较为直接地获悉其对中国文学外译 中若干问题的思考。

2012年8月, 第二届汉学家文学翻译国际研讨会在北京举行, 核心话题 是 “全球视角下的中国文学翻译”。会上, 她发言的题目是《中国文学西 译存在的问题及建议》。她首先提出 “网络的普及使得 “景观文化” 日益盛 行，笔译面临着前所未有的挑战。”之后，她强调原始版本的选择和目的语 写作能力的重要性, 认为 “无论古典文学还是现当代文学作品都存在这个问 题”。这里有必要补充菲萨克（2012）在其后的一篇论文及与笔者的谈话中 提及的观点。她认为不少中国现代作家都不止一次地修改过他们的作品, 巴 金尤具代表性，他对《家》曾做出过累计达 14000 处大小不等的改动, 形成 1933、1953、1958三个重要版本。多数外译本均依据1958版或其简写本，几 乎没有任何一个译本源自《时报》连载版（1931.4.18-1932. 5. 22, 原名《激 流》) 或开明书局1933年5月出版的单行本。她表示以后有时间, 要依据1933 年的初版本重新翻译《家》这部销量极高的现代文学作品。可见, 菲萨克 （2012）不仅重视源语版本，同时更强调原始版本的价值。发言中，她还特 别指出 “译者把握西班牙语的能力是创作优秀的汉译西作品的必要条件。 她认为其重要性甚至不亚于译者的汉语水平，必须引起足够的重视。

第三届会议上，她发表了《汉西不同的故事叙述方式》的讲话。话题聚 焦于文学翻译中的准确性与流畅性问题, 因为准确与流畅有时候很难兼顾。 在翻译实践中，菲萨克（2015）敏锐发现，中国当代文学由于突破了 “为工 农兵服务” 的政策制约，作家们富有主观创造性和实验性的写作方式使得文 学作品更加丰富多彩, 但同时也给文学翻译工作带来了极大的挑战。之前翻 译鲁迅、巴金、钱钟书作品时，“贴近原文” 难度还不是很大。当代作家风 格的独特性与多样性促使她思考怎样在目的语中呈现出不同的风格。为此, 她着眼于中西语言关于时空表达、语境语序等诸多方面的差异, 提出了翻译 中需要注意的问题。西语时间差异体现在动词变位上, 空间词较少, 对于语 境的依赖性小，语序依主次排列。相反，汉语时间表达较为模糊，表示空间 变化和差异的词非常多, 对语境的依赖性较高, 同时还有很多言外之意, 语 序排列与西语差异较大, 往往限定词在前, 这些对西语读者来说都是较难理 解的。所以, 翻译中文作品的译者, 必须对此做出变通, 同时, 还要熟悉中 西方文化和表达习俗, 这样才 “有能力用西班牙语或其他语言比较忠实地表 现原文的写作风格”，“既不能背叛原文，也不能背叛目的语”。

第四届会议, 她针对具体问题, 提出了关于中文人名和地名《可译与不 可译》的观点（下文详述）。 
由此可见, 翻译前, 她注重版本选择, 强调源语版本及其原始版本的 重要性; 翻译时, 重视译者的目的语表达能力, 认为它与译者的中文理解 力、两种文化和习俗的掌握都是不可或缺的。正如 Eugene A. Nida和Charles R.Taber（1969）所言： “翻译是用最恰当、自然和对等的语言从语义到 文体再现源语的信息。”在翻译实践中, 菲萨克注重总结和积累经验, 从 内容到风格, 不断提高译作的质量。其翻译思想比较接近霍克思 (David Hawkes，1973），即 “译者要 “忠实” 的对象是多元的一一既要忠实于作 者, 又要忠实于读者, 还要忠实于艺术。”

\section{3. 翻译方法}

\section{1 直译与转译之取舍}

这里所谓直译和转译, 指的是中国文学译入西班牙的两种途径, 是根据 源语言和译入语的关系而做出的区分。直译即直接从中文译入西班牙语; 转 译即经由第三种语言间接将中国文学作品译入西班牙。“转译是二十世纪中 国文学在西班牙的主要译入方式, 多于直译; 转译说明了在二十世纪西班牙 处于边缘地位。”（Marín，2012）当然，这是就整体而言。但是，在整体并 不乐观的情形下，也存在着某些可喜的景况。

自1982年首次发表汉西翻译作品伊始, 菲萨克（2012）所有的译作均 选自中文原版。在第二届文学翻译国际研讨会上, 她说 “中国现当代文学作 品的西语翻译很多译自英语或法语文本, 翻译质量不高。” 确实如此, 因为 从根本上讲, 翻译就是对异域文化及其意识形态的输入，对本土价值体系而 言, 是一种外来文化的渗透, 它意味着对本土文化的考验, 也可能伴之以某 种程度的破坏和颠覆。转译自英法版本的中国文学作品, 很难原汁原味地再 现源语文本的语言特色、风格特征和文化意蕴。因为它已经按照英/法语读 者的接受偏好做出了程度不同的处理, 无论语言还是内容, 都既可能有保留 或突出东方异域风情的异化处理, 也可能有极力贴近目的语文化习俗和审美 习惯的归化式变通。我们知道, 从源语文本到目的语文本, 译者要同时在语 义、逻辑和审美三个平面上工作，既有认知心智、又有审美心理的投入。译 者对源语文本语义的把握常常需要逻辑的调整、修正与校正, 再经过审美的 判断、优选和定夺, 才能最后赋形为译文。可谓 “方寸之地, 举步维艰”。 （刘宓庆，1996）虽说翻译过程中的增删改变在所难免, 但条件具备的话, 还是以选择源语文本最为合适。译者只有从中文版本直译，才能获得最深切 的感受, 体会中西思维、语言表达及文化背景的差异, 进而确保译本的准确 无误, 最后才谈得到乑于完美。

\section{2 直译与意译之平衡}

发言中, 菲萨克（2012）坦承：“译者在对直译和意译做出选择的时 候, 折衷往往是一个好办法。”认为翻译出中文原文的风格特点, 在很多情 
达西安娜・菲萨克对中国现当代文学作品 的译介研究
Taciana Fisac's Translation of Chinese Modern and Contemporary Literature

况下不大可能, 只是一种理想境界。纵观菲萨克的译作，多数情况下，她坚 持直译为主, 意译为辅。

这里的 “直译” 不同于上文, 指的是译文既忠实原文内容, 又符合原 文的语言与文体结构。这是由于不同民族对客观事物的感受及社会经历、情 感阅历等方面有某些相似之处，不同语言也有少量相同或近似的表达方式， 客观存在着可以互译的表达方式，它们传达着相同的文化信息，字面意义、 形象意义，乃至隐含意义都基本相同或相近。比如阎连科在《四书》开端写 下: “大地和脚, 回来了。” 菲萨克 (2016) 将其译为 “La madre tierra y los pies habían regresado.”这就体现出中西人民对大地的等值看法, 都内涵 着大地母亲的意指。

“意译”则从意义出发, 只要求将源语文本的大意表达出来即可, 不需过 分注重细节, 但要求译文自然流畅。由于文化因素的影响, 在翻译时无法保 留原文的字面意义和形象意义，便将源语文本中的形象更换成另一个目的语 读者所熟悉的形象, 从而传递原文的语用目的, 译出隐含意义。汉西两种语 言不同的发展历史，以及不同的民族文化、风俗习惯造成了两个民族各不相 同的语言与表达方式。因此, 菲萨克强调译者必须对两种语言文化及文学表 达有较好的认识和较高的应用水准, 尤其是目的语的修养。因为每种语言都 有自己的表达习惯和言外之意, 在深刻领会原著精神实质的前提下, 无法逐 字翻译时，便要不拘泥小节，创造性的表达原著思想，但不可添枝加叶、改 变原作的风格。她认为最好的做法是冷处理, 即先将直译自中文原版的译文 搁置一段时间，让思绪出离作品所营造的语境，待一定时间的沉淀后，完全 以目的语读者身份品读译本, 这时, 将那些不符合目的语读者思维和表达习 惯与习俗的语句重新写过。这样, 既保留了原意, 又是地道的目的语表述。 当然, 她同时也强调在全球视角下，译者更应保持对文学差异性的敏感，就 中国文学而言, 这种差异性正是它的活力所在。这里, 我们仍以《四书》为 例, 阎连科对昼夜分离的描写是: “光是好的, 神把光暗分开。称光为昼, 称暗为夜。有晚上, 有早上。这样分开。暗来稍前, 称为黄昏。黄昏是好 的。”译文是“La luz era buena y Dios separó la luz de las tinieblas. Llamó a la luz «día» y a las tinieblas, «noche». Y hubo ocaso y amanecer. Así se separaron. Al momento antes de llegar la oscuridad lo llamó «atardecer». El atardecer era bueno.”(菲萨克，2016) 我们看到译文明显长于原文，这 里菲萨克采用了西语版《圣经 - 旧约》的叙述方式，既符合西语读者的表达 习惯和接受心理, 也体现了阎连科在《四书》中刻意使用的叙述方式之一, 即“圣经式”语句。

\section{3 原著风格与目的语表达之卙酌}

在第三届汉学家文学翻译国际研讨会上, 菲萨克（2015）做了 “译者 是 “叛徒” ? ” 的大会致辞。其中有这样一句: “我也不敢轻易说我不是 叛徒。因为我们绝大多数人在翻译过程中都有意无意地改变过原作的叙事方 
式、语言风格等等。”作为汉学家、翻译家的菲萨克, 随着其翻译经验的丰 富, 翻译思想和方法也愈趋成熟。虽然她说 “译者是 “叛徒” ”, 但仔细阅读 她的译作，不难发现她的个人风格是“忠于原文、忠于读者、忠于艺术”的， 她的译本准确、流畅，符合目的语读者的表达习惯和审美情趣。

我们以实例来感受其在勘酌、把握两种语言的不同表达时所耗费的心 神。笔者选取最能代表其现阶段翻译水准和风格的译作（毕飞宇的《大雨如 注》和铁凝的《飞行酿酒师》) 中的句子试做分析:

（1）哎，这个米歇尔也真是，就一个小时的英语对话，非得弄到足球 场上去。

(2) ¡Vaya!, esta Michelle era realmente un caso.Para una hora de conversación de inglés había tenido que irse al campo de fútbol.

(3) 可大姚还是越想越气, 越气越委屈。他对着查无踪影的女儿喊了 一声：“我有钱！你老子有钱哪!”

终于喊出来了, 可舒服了, 可过了瘾了。

一个过路的小伙子笑笑, 歪着头说: “我可全听见了哈。”

(4) Pero cuanto más pensaba en ello, más enfado sentía, y cuanto mayor el enfado, mayor la injusticia. Gritó tras el rastro ya inexistente de su hija: «iTengo dinero!, ¡tu padre tiene dinero!»

Había terminado por soltarlo, se había sentido bien,y se había quedado tan ancho.

Un joven que cruzaba la calle sonrió, ladeó la cabeza y dijo: «Ja, que lo he oído todo».

（5）华灯初上，车灯们也哗啦啦亮起来。城市的灯火是这样密集、晶 莹如香槟的泡沫：这个形容的发明权不属于无名氏，他是从多少年前读过的 一本外国小说里搬来的。

(6) Cuando comienzan a brillar las luces se encendían también los fogonazos de los faros. Las luminarias de la ciudad eran tan concentradas y cristalinas como la espuma del champán. La patente de invención de este calificativo no era patrimonio del señor X,la había tomado de una novela extranjera que había leído hacía varios años.

（7）这让他突然很想和从前的那个老同学 “高原红” 通个电话，他很 想听“高原红”再对他说一句“饿喝不惯，饿实在是喝不惯”。

(8) Aquello hizo que de repente le entraran ganas de llamar por teléfono a su antiguo compañero de estudios apodado el Altiplano Rojo;quería escuchar de nuevo aquella frase suya:«¡Yo no aguanto este sabor! ¡Es realmente intragable!» 
达西安娜・菲萨克对中国现当代文学作品 的译介研究
Taciana Fisac's Translation of Chinese Modern and Contemporary Literature

其中涉及到人名、绰号、成语、方言、时间、心理、语气词等的处理。 语气词，如（1）中的 “叹”，（3）中的 “哈”，译本中相应词语的发音、 语气（[2]中的;Vaya!）、位置（[4]放在句首的Ja）都有一定的变化，这样 更符合西语读者的表达和接受习惯。人名，如（5）中的 “无名氏”，（7） 中的 “高原红”, 翻译时采用了不同的策略, 无名氏按未知数通常的表示方 法写成X，高原红则直译为Altiplano Rojo，即 “高原” +“红”。成语、方言， 如（5）中的 “华灯初上” 和（7）中的 “饿”，在西语中完全没有对应的表 达, 于是, 译文采用倒装句的方式传达了相近的语义Cuando comienzan a brillar las luces se encendían también los fogonazos de los faros (当开 始发光时，一盏盏的灯陆续打开，还有闪㷧的车灯。意思接近原文的 “华灯 初上，车灯们也哗啦啦亮起来”），这既涉及到西语表达习惯，也有指向、 复数、自复等语法, 尤其重要的是中西语言基于思维习惯而产生的截然不同 的表述方式。中国人习惯先提出话题, 然而加以解释或描述, 即 “话题, 解 释”。但是西语没有类似的思维习惯和表达方式, 于是汉语的 “华灯初上, 车灯也哗啦啦亮起来” , 在西语中是没有停顿的, 而且描述方法和语序均不 同于原文。汉语方言 “饿” 则直接译作Yo, 即 “我”。表达心理变化的特殊 词语，各语种差别较大，如（1）中的 “这个米歇尔也真是” ，翻译时则变成 了 “esta Michelle era realmente un caso”，表示不满的程度不及汉语的“也 真是”, 但是也含有真是个问题之意。西语动词因人称、时间不同而有诸多 变位, 汉语动词却没有词形变化, 翻译时译者必须根据上下文语境, 准确变 位。如（3）中的 “(大姚）终于喊出来了, 可舒服了, 可过了瘾了。一个过 路的小伙子笑笑, 歪着头说” , 西译版是“Había terminado por soltarlo, se había sentido bien,y se había quedado tan ancho. Un joven que cruzaba la calle sonrió, ladeó la cabeza y dijo"大姚的动作使用的动词变位是陈述式过 去完成时，过路的小伙子则使用了陈述式未完成时和简单过去时，“过瘾” 则意译为内心宽阔即舒畅了。

即便只是个别语句的对比分析，亦足以见出菲萨克在如何使目的语文本 更贴近原著风格方面颇下了一番功夫，寻找准确的词语、重新创造合适的措 辞, 以确保她的译本在忠实原文的基础上, 更贴近西语读者的接受心理, 也 更具有文学气息。

\section{4 人名地名等专有名词的汉西对接}

中西文化的差异体现在诸多方面, 这使得译者在翻译中国文学作品时, 不得不如娜佳 (2012) 所言, 要 “考虑到以下三种读者的兴趣取向：一般的 读者、汉学专家读者和文学专家读者 (后者不一定会说汉语, 也不一定对中 国文化和文学很熟悉, 但是他/她研究文学发展过程的普遍规律, 对世界各 国文学都感兴趣。）”于是, 面对为数众多、不懂汉语的读者, 即便是那些 简洁、美妙、寓意丰富的中国人名、地名的翻译, 译者也时常左右为难, 踌 躇再三。 
2016年8月第四届汉学家文学翻译国际研讨会在长春举行, 菲萨克以 《可译与不可译》为题, 结合翻译实践, 提出了中文人名、地名的翻译问 题。菲萨克 (2017) 认为, “既然作家有意选择那些耐人寻味的人名和地 名, 译者就不应简单采用汉语拼音, 而应使用富有新意、含义相近的目的语 名词。”“汉学家、翻译家都必须重新评估并认真考虑汉语拼音在人名地名 翻译中的局限性问题, 以准确传达作者意图为旨归, 出色完成文学翻译的任 务。只有这样，才能更加有效地推进中国文学作品在世界范围内的认知度及 欣赏度。”她认为某些人名、地名的拼音直译不足以展现作家的创造力, 因 为它完全取消了名字的象征意义及多重含义。她以巴金的《家》、莫言的《红 高粱》和《丰乳肥臀》、阎连科的《受活》为例, 提出了自己的看法。一类 是如觉新、觉民、觉慧，来弟、招弟、想弟、盼弟、念弟、求弟，玉女、金 童这样有寓意又有文化特点的名字，一类是如茅枝婆、菊梅、桐花、槐花、 榆花和么蛾儿这样以花为名的情形。前一种情况既有中国人命名时的家族辈 分传统, 又有特定的文化寓意, 昭示着某种时代需求和个人需求。后一种则 是中西共存的取名方式。面对这些情况，她提出有的，譬如《受活》中的几 个女性名字可以直接译成西语花名, 易读又好记。而面对《家》和《丰乳肥 臀》里那些中国传统性强、意蕴丰富的名字, 既然西班牙语没有这样的字母 组合、读起来很别扭, 那是不是可以考虑从意义方面给以重新命名。她说 30 年前, 她翻译《家》时, 觉得Juéhui这样的组合西班牙语里没有, 很难读出 来，她就改写成Chuehui。但她问了一些读者，大部分人都说 “这三个人的 名字太像，看小说的时候分不清楚谁是谁”。真如严复（1981）所云 “一名 之立, 旬月趾䠎”。

地名的翻译也存在同样的问题。她建议一是采用每种语言普遍使用的说 法, 如 “北京” ，西语是Pekin。二是意译，如 《受活》所涉及的地名, 意义 丰富且复杂。目前, “受活庄” 西译本意译为Buenavida, 即好的生活。菲 萨克认为这样的翻译比较贴切, 因为它至少比直接写作好得多。

文学国际化的历程一方面彰显着源语文学创作的成就, 一方面见证着 目的语读者了解源语国的需求, 同时, 也从一个侧面反映着国际战略与国际 关系的发展变化。谢天振（2013）认为，“不同民族、国家之间文化交流的 流向是有其内在规律的, 总体上总是从强势文化流向弱势文化, 这样的例子 在古今中外翻译史上可以说比比皆是。这也在一定程度上解释了为什么我们 对西方文化、文学的译介品种和数量都远远超过西方国家对我们国家文化、 文学的译介品种和数量。”可喜的是, 近年来, 随着中国整体实力和国际影 响力的增强, 中国文学文化的输出也超越了以往任何时期。尤其难得的是我 们拥有越来越多像菲萨克这样的汉学家、翻译家, 他们把中国文学看作个人 生活的一部分, 并且是 “生活中特别重要的一部分” 。对中国文化和中国文 学的痴情, 令他们甘愿坐在冷板登上, 年复一年地穿梭于两种文化和语言之 间，阅读、精选、翻译并打磨手中的译本（刘宓庆，1996）。他们希望能尽 一己之力, 让读者看到真正的中国精神, 去了解自己无法亲自识见的遥远的 中国。正因如此, 菲萨克总是选择源语文本, 她既重视译者对源语文学作品 
达西安娜・菲萨克对中国现当代文学作品 40 的译介研究
Taciana Fisac's Translation of Chinese Modern and Contemporary Literature

的整体把握与鉴赏能力, 又特别强调译者目的语的文学表达与文化传递水 准; 既注重对源语文学作品叙事方法与风格的尊重, 也顾念目的语读者的接 受心理与表达方式。在直译与意译、异化与归化间寻求平衡, 以期实现 “忠 于作者，忠于读者，忠于艺术”的目标。

李素 (2012) 认为, “翻译文学的好坏不在于其学术意义上的确切, 也 不在于翻译过程的某种层次, 而在于翻译作品的接受是否成功。只有踏踏实 实地立足在他国文化的翻译作品, 也就是和他国文学打通了桥梁的文学翻译 作品, 才算是真正的被翻译成他国语言, 被纳入到他国文化中。”我们有理 由相信, 借助优秀的汉学家翻译家们的辛勤耕耘, 他们解读的中国故事, 即 外译中国文学作品, 经历漫长的过程后, 中国文学会以越来越真实和完整的 面貌出现在海外读者面前。

\section{参考文献}

Ba, J.,1989. La familia Kao. Traducción de Taciana Fisac. Madrid: Ediciones SM.

Bi, F. Y., 2017. Caían chuzos de punta. Traducción de Taciana Fisac. Leersenda de papel, (2): 2.

David, H., 1973. The Story of the Stone. Vol. 2, Penguin Books.

Eugene, A. N., \& Charles, R. T., 1969. The Theory and Practice of Translation. Leiden: E. J. Brill.

Gao, X. S., 1989. Chen Huansheng va a la ciudad. Traducción de Taciana Fisac. Calle Mayor, (3): 60.

Maialen Marín, L., 2012, Mediación, recepción y Marginalidad: Las Traducciones de Literatura China Moderna y Contemporánea En España. Universitat Autónoma de Barcelona: 52-53.

Qian, Z. S., 2009. La fortaleza asediada. Traducción del chino de Taciana Fisac. Barcelona: Editorial Anagrama, S.A.

Taciana, F., 1982. 2 cuentos chinos, que presenta Taciana Fisac. Nuevo Indice, (3): 18.

--, 1989. Notas acerca de la vida y obra de Ba Jin. Ba Jin. La familia Kao, Traducción de Taciana Fisac. Madrid: Ediciones SM.

--, 2012. Anything at Variance with it Must Be Revised Accordingly: Rewriting Modern Chinese Literature During the 1950s. The China Journal, 67 (1): 131-148. 
--, 2016. Para leer Los cuatro libros. Yan, L. K., Los cuatro libros. Traducción del chino de Taciana Fisac. Barcelona: Galaxia Gutenberg: 7-13.

Tie, N., 2016. El enólogo volador. Leer-senda de papel. Traducción de Taciana Fisac, (1): 2.

--, 1989, La blusa roja sin botones. Traducción de Taciana Fisac. Madrid: Ediciones SM: 5-8.

Yan, L. K., 2016. Los cuatro libros. Traducción del chino de Taciana Fisac. Barcelona: Galaxia Gutenberg.

达西安娜·菲萨克, 2012, 中国文学西译存在的问题及建议 [M]。中国作家协 会外联部编, 翻译家的对话 (二)。北京: 作家出版社。

达西安娜·菲萨克，2015，汉西不同的故事叙述方式 $[M]$ 。中国作家协会外联 部编, 翻译家的对话（三）。北京：作家出版社。

达西安娜·菲萨克, 2015, 译者是“叛徒”? [M]。中国作家协会外联部编. 翻译 家的对话（三）。北京：作家出版社。

达西安娜·菲萨克, 2017, 可译与不可译 $[\mathrm{M}]$ 。中国作家协会外联部编, 翻译 家的对话（四）。北京：作家出版社。

贾植芳, 2003 , 序一, 参见谢天振, 译介学 $[\mathrm{M}]$ 。上海: 上海外语教育出版社。 李素, 2012, 翻译中国还是翻译文学: 捷克的传统与今天的经验（二） [M]。 北京：作家出版社。

刘宓庆, 1996, 翻译的美学观 $[J]$ 。外国语, (5) : 2 娜佳, 2012, 从乌克兰视角看中国文学翻译 [M]。中国作家协会外联部编, 翻 译家的对话。北京：作家出版社。

王向远，2015，中国翻译思想的历史积淀与近年来翻译思想的诸种形态 $[\mathrm{J}]$ 。 广东社会科学, (5) : 151-152。

谢天振, 2013 , 换个视角看翻译一一从莫言获诺贝尔文学奖谈起 $[\mathrm{J}]$ 。东方翻 译 (1) : 4-8.

严复, 1981, 译例言, 天演论 [M]。北京：商务印书馆。 
